\title{
Sonography of the Lateral Antebrachial Cutaneous Nerve With Magnetic Resonance Imaging and Anatomic Correlation
}

\author{
Mary M. Chiavaras, MD, PhD, Jon A. Jacobson, MD, Lisa Billone, BS, Jason Michael Lawton, \\ Jeffrey Lawton, $M D$
}

Received October 22, 2013, from the Department of Diagnostic Imaging, McMaster University, Hamilton General Hospital, Hamilton, Ontario, Canada (M.M.C., L.B.); and Departments of Radiology (J.A.J.) and Orthopedic Surgery (J.M.L.J.L.), University of Michigan, Ann Arbor, Michigan USA. Revision requested November 18, 2013. Revised manuscript accepted for publication November 25, 2013.

We thank Glen Oomen for preparation of the color illustrations.

Address correspondence to Mary Chiavaras, MD, PhD, Department of Diagnostic Imaging, Hamilton General Hospital, 237 Barton StreetE, Hamilton, ON L8L 2X2, Canada.

E-mail:chiavamm@mcmaster.ca

\section{Abbreviations}

$L A B C N$, lateral antebrachial cutaneous nerve; MRI, magnetic resonance imaging

doi:10.7863/ultra.33.8.1475
Objectives - Abnormalities of the lateral antebrachial cutaneous nerve (LABCN) are associated with antecubital elbow conditions, such as distal biceps brachii tendon tears and traumatic cephalic vein phlebotomy. These can lead to lateral forearm, elbow, and wrist symptoms that can mimic other disease processes. The purpose of this study was to characterize the sonographic appearance of the $\mathrm{LABCN}$ using cadaveric dissection and retrospective analysis of sonographic examinations of symptomatic patients with magnetic resonance imaging correlation.

Methods - For the first part of this study, a cadaveric elbow specimen was examined, and sonography was performed after dissection to identify the LABCN. Subsequently, 26 elbows in 13 patients with $\mathrm{LABCN}$ abnormalities were identified with sonography and retrospectively evaluated to characterize the appearance of the LABCN in both symptomatic and asymptomatic elbows.

Results - The symptomatic LABCNs showed fusiform enlargement, increased echogenicity, and loss of the normal fascicular echo texture. The mean cross-sectional area of the symptomatic nerves was $12.0 \mathrm{~mm}^{2}$ (range, $6.1-17.2 \mathrm{~mm}^{2}$ ), with a maximum thickness of $3.5 \mathrm{~mm}$ (range, $2.3-5.9 \mathrm{~mm}$ ), compared to $3.3 \mathrm{~mm}^{2}$ (range, $1.9-5.2 \mathrm{~mm}^{2}$ ), with a maximum thickness of $1.3 \mathrm{~mm}$ (range, $0.9-2.2 \mathrm{~mm}$ ), in the contralateral normal elbows.

Conclusions - The close proximity of the LABCN to the distal biceps tendon and the cephalic vein makes it vulnerable to compression and injury in the setting of distal biceps tendon tears and traumatic phlebotomy, which may cause nerve enlargement and increased echogenicity. Awareness of the location and appearance of the LABCN on sonography is important for determining potential causes of lateral elbow and forearm pain.

Key Words - elbow; magnetic resonance imaging; musculoskeletal ultrasound; nerve; sonography

$\mathrm{T}$ he lateral antebrachial cutaneous nerve ( $\mathrm{LABCN})$, or lateral cutaneous nerve of the forearm, is a terminal sensory branch of the musculocutaneous nerve, lying adjacent to both the distal biceps brachii tendon and cephalic vein within the antebrachial region of the elbow (Figure 1$).{ }^{1}$ The close proximity of this nerve to these structures makes it susceptible to compression or injury secondary to trauma and inflammatory processes involving the anterior elbow (Figure 2$).{ }^{2}$ Compression of the LABCN typically presents as pain and dysesthesia along the lateral forearm, which can extend 
from the elbow to the wrist. This condition may mimic other causes of lateral elbow pain, such as lateral epicondylitis and radial tunnel syndrome, and can masquerade as a wrist disorder. ${ }^{3,4} \mathrm{~A}$ clear understanding of the location and imaging appearance of the $\mathrm{LABCN}$ is important for accurate diagnosis.

Lateral antebrachial cutaneous nerve entrapment neuropathy has been described in the orthopedic and plastic surgery literature, resulting from distal biceps brachii tendon tears, ${ }^{5,6}$ distal biceps brachii tendon repair, ${ }^{7}$ cephalic vein phlebotomy, ${ }^{8-12}$ arm positioning during prolonged

Figure 1. Anterior elbow illustration showing the LABCN (yellow arrow) as a continuation of the musculocutaneous nerve (black arrow) after it pierces the deep fascia (white arrow) just proximal to the elbow joint. Note the proximity to the distal biceps brachii tendon (black arrowhead) and cephalic vein (blue arrow). Compression or injury of the LABCN may lead to pain and dysesthesia along the anterolateral forearm (red area).

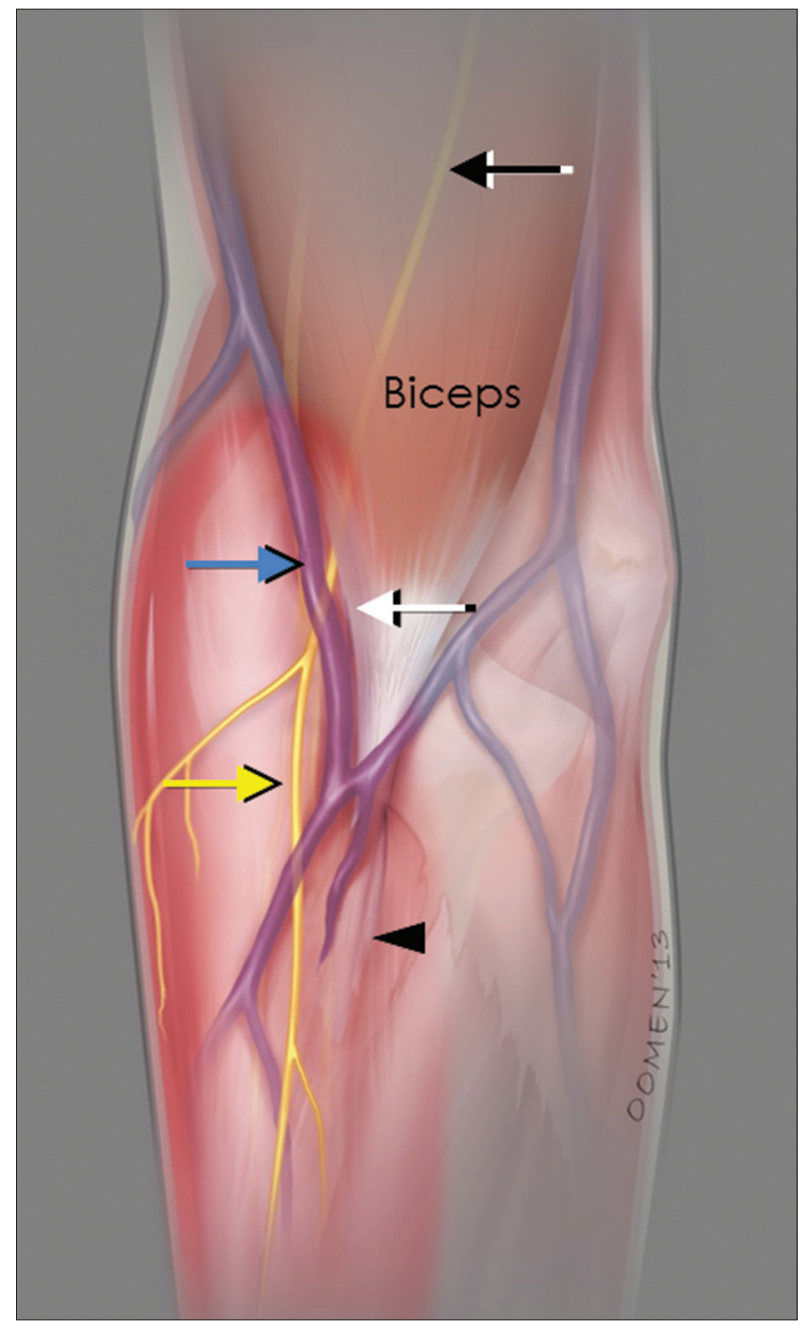

anesthesia for knee surgery, ${ }^{13}$ and pull-up exercises during military training camp. ${ }^{14}$ Despite the well-recognized clinical entity, there is little published regarding the normal and abnormal sonographic appearances of the LABCN. Sonography of the musculocutaneous nerve has been described; however, detailed assessment of the terminal $\mathrm{LABCN}$ cutaneous branch was not performed. ${ }^{15} \mathrm{~A}$ recent study, examining mimickers of lateral epicondylitis, reported that the $\mathrm{LABCN}$ is identifiable on both sonography and magnetic resonance imaging $(\mathrm{MRI})^{16}$; however, a systematic and quantitative analysis of both the normal and abnormal appearances of the LABCN in a larger number of patients was lacking.

In our clinical practice, sonography is often used to evaluate the elbow for various disorders, including characterization of distal biceps brachii tendon tears and general assessment of lateral elbow pain. We have observed abnormalities of the LABCN, particularly in patients with distal biceps brachii tendon disorders who describe pain and sensory changes involving the lateral forearm. The purpose of this study was to characterize the normal and abnormal $\mathrm{LABCN}$ as seen on sonography using cadaveric dissection and retrospective analysis of sonographic examinations of symptomatic patients with MRI correlation.

\section{Materials and Methods}

\section{Cadaveric Dissection and Sonographic Evaluation}

After approval by the Anatomical Donations Department, 1 fresh, nonembalmed elbow specimen was dissected by a fellowship-trained orthopedic surgeon (15 years of elbow surgery experience) to visualize the LABCN. The skin, subcutaneous tissue, and fascia were removed to expose the distal biceps tendon and LABCN (Figure 3). The specimen was then placed in a water bath and imaged with sonography by a musculoskeletal fellowship-trained radiologist (18 years of musculoskeletal sonography experience) using a 12-MHz linear transducer (iU22; Philips Healthcare, Bothell, WA). Specifically, the LABCN was imaged in short and long axes. The sonograms of the specimen were reviewed in consensus by 2 fellowship-trained radiologists ( 15 and 3 years of experience), and the LABCN was characterized with respect to location, fascicular appearance, and echogenicity.

\section{Retrospective Sonographic Evaluations of Patients}

Research Ethics Board approval was obtained for this aspect of the study. All elbow sonographic reports from the primary author's institution over an 18-month period were reviewed to identify patients in whom the $\mathrm{LABCN}$ was 
described as abnormal. Sonographic examinations of the elbow were performed as part of routine patient care by 1 of 2 fellowship-trained musculoskeletal radiologists ( 3 years and 10 years of experience) using both 12 - and $17-\mathrm{MHz}$ linear transducers (iU22; Philips). Routine sonography included static images and cine clips saved to a picture archiving and communication system. The use of color or power Doppler imaging was at the discretion of the radiologist, as well as comparison imaging of the contralateral elbow.

Sonograms were then retrospectively reviewed to characterize the LABCN by one of the above fellowshiptrained musculoskeletal radiologists (3 years of experience). On sonograms in the transverse plane relative to the humerus, the $\mathrm{LABCN}$ was identified just lateral to the distal biceps brachii tendon as a small oval structure with hypoechoic fascicles and surrounding hyperechoic connective tissue, characteristic of a peripheral nerve (Figure 4).${ }^{17}$ The cephalic vein may be visualized, when not compressed during ultrasound scanning, coursing from lateral to medial and crossing superficial to the $\mathrm{LABCN}$ at the level of the antebrachial fossa. On an imaging workstation, the largest dimension of the $\mathrm{LABCN}$ when imaged in the short axis was measured using digital calipers and recorded. Similarly, the crosssectional area of the LABCN was calculated in the circumferential trace mode. Other sonographic characteristics of the LABCN that were recorded included echogenicity

Figure 2. Anterior elbow illustrations showing fusiform enlargement of the LABCN (yellow arrows) secondary to a partial tear of the distal biceps brachii tendon (A) with associated hematoma (white arrow) and traumatic venipuncture of the cephalic vein (B, blue arrow).

A

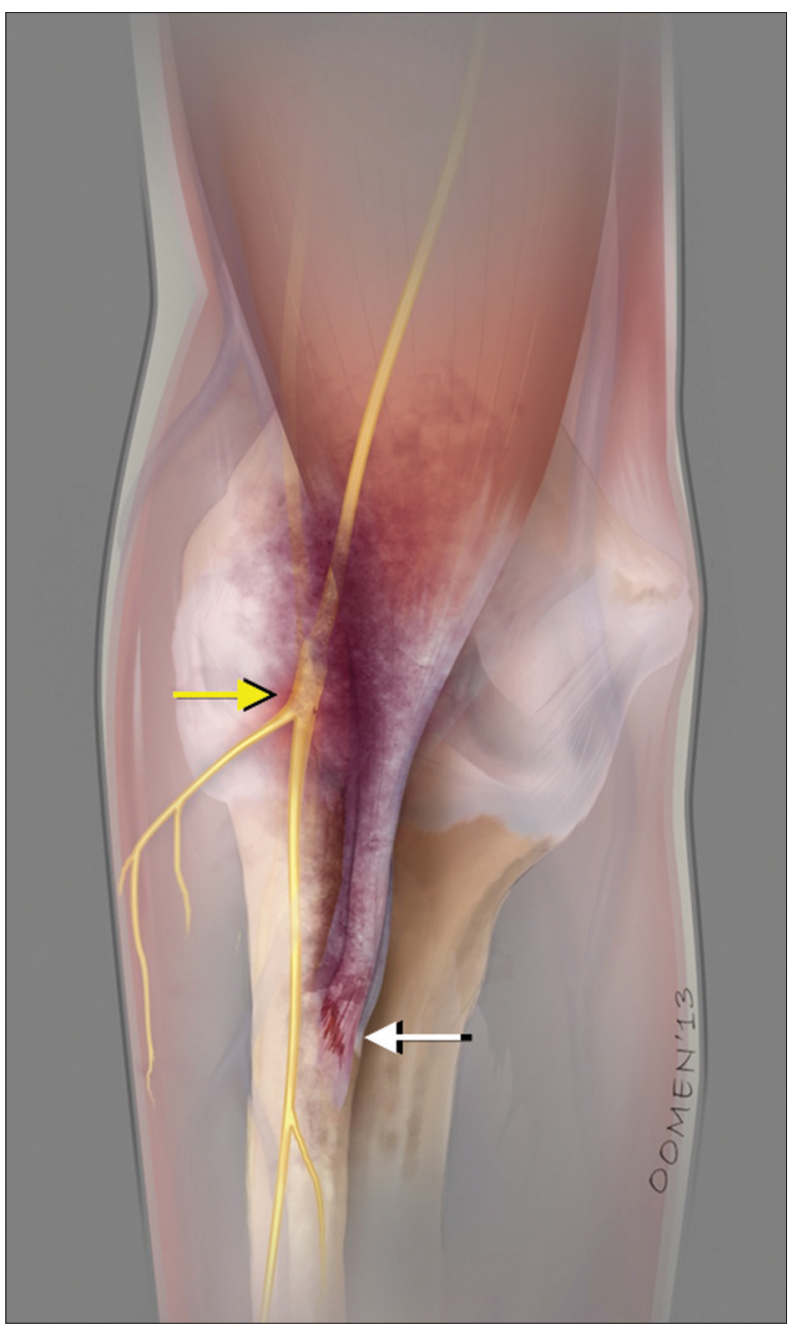

B

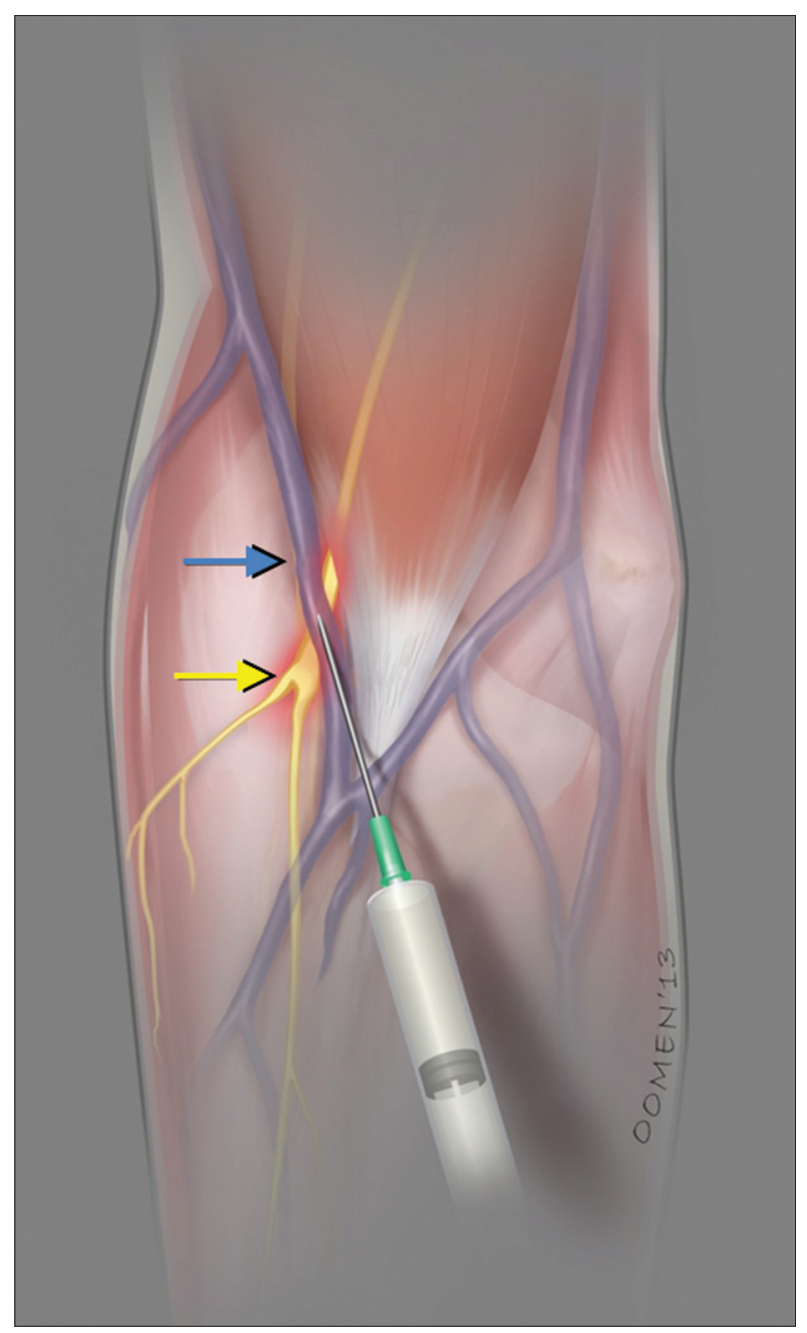




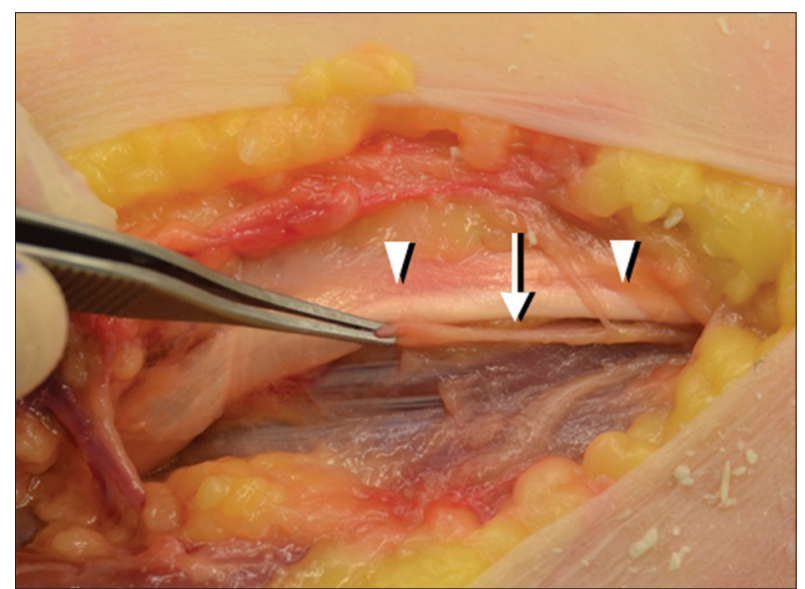

A

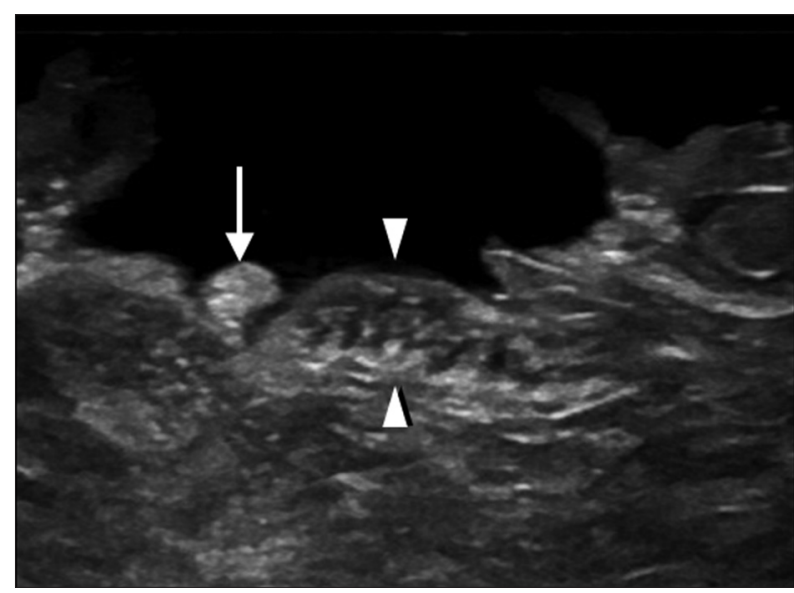

B

Figure 3. Images from a 65-year-old woman with a normal LABCN (cadaveric specimen). A, Dissected anterior elbow specimen showing the LABCN (white arrow) located just lateral to the distal biceps brachii tendon (white arrowheads). B, Sonography over the anterior elbow of the cadaveric specimen in the transverse plane, performed in a water bath after removal of the superficial subcutaneous soft tissues, showing the LABCN (white arrow) in the short axis adjacent to the distal biceps brachii tendon (white arrowheads).

(normal fascicular pattern, abnormal hypoechoic appearance with loss of the fascicular pattern, or abnormal hyperechoic appearance with loss of the fascicular pattern). The contralateral LABCN was similarly characterized when imaged. Other diagnostic information regarding the anterior elbow was also recorded from the prospective sonographic reports, such as status of the biceps brachii tendon and adjacent abnormalities. Patients' clinical records and surgical reports (if applicable) were also reviewed. Correlations with MRI were made when available.

\section{Results}

The initial search of consecutive elbow sonographic examinations over 18 months yielded 137 examinations in 130 patients ( 7 cases were complete bilateral elbow examinations). Among these 137 examinations, the LABCN was described as abnormal in 13 patients (all male; mean age, 51 years; range, $30-86$ years). The right and left elbows were affected in 6 and 7 patients, respectively. Color Doppler flow was assessed in 11 patients, and the contralateral asymptomatic elbow was evaluated for comparison in each of the 13 patients.

At sonographic characterization (Table 1), the mean abnormal LABCN measurement was $3.5 \mathrm{~mm}$ at its maximum thickness (range, $2.3-5.9 \mathrm{~mm}$ ), and the mean cross-sectional area was $12.0 \mathrm{~mm}^{2}$ (range, $6.1-17.2 \mathrm{~mm}^{2}$ ). In each case, the affected nerve segment was abnormally hyperechoic with loss of its fascicular pattern (Figures 5-7).
There was evidence of increased flow on color Doppler imaging in 1 patient (Figure 7). The mean contralateral asymptomatic LABCN measurement was $1.3 \mathrm{~mm}$ (range, $0.9-2.2 \mathrm{~mm}$ ), and the mean cross-sectional area was $3.3 \mathrm{~mm}^{2}$ (range, 1.9-5.2 $\mathrm{mm}^{2}$ ), with each nerve showing a normal fascicular pattern on sonography (Figures 5-7).

Among the 13 patients, sonographic reports described distal biceps brachii tendon tears in $85 \%$ (11 of 13 ): 5 partial-thickness tears (Figure 5) and 6 full-thickness tears (Figure 6). Two of these patients had MRI, and 7 had surgical confirmation. One patient had a chronic postopera-

Figure 4. Sonogram of the anterior elbow in the transverse plane, just proximal to the elbow crease, in an asymptomatic volunteer showing a hypoechoic $L A B C N$ with a normal fascicular echo texture (black arrowheads) in the short axis located lateral to the biceps tendon (BT; white arrowhead). The brachial artery ( $\mathrm{A}$ ) and median nerve (MN; black arrow) are located medially.

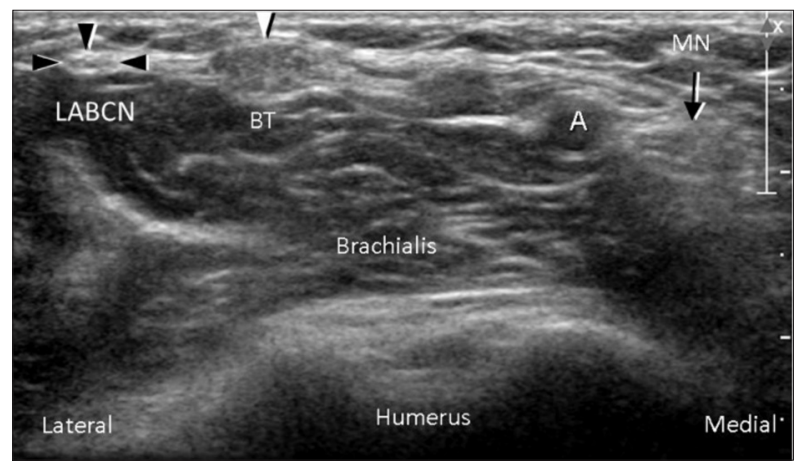


Table 1. Demographic Characteristics and Sonographic Findings for the 13 Patients With LABCN Abnormalities

\begin{tabular}{|c|c|c|c|c|c|c|c|c|c|}
\hline \multirow[b]{2}{*}{ Patient } & \multirow[b]{2}{*}{ Age, y } & \multirow[b]{2}{*}{ Sex } & \multirow[b]{2}{*}{$\begin{array}{c}\text { Biceps Brachii } \\
\text { Tear }\end{array}$} & \multicolumn{3}{|c|}{$\begin{array}{c}\text { Abnormal } \\
\text { Symptomatic LABCN }\end{array}$} & \multicolumn{2}{|c|}{$\begin{array}{c}\text { Normal } \\
\text { Contralateral LABCN }\end{array}$} & \multirow[b]{2}{*}{$\begin{array}{c}\text { Surgical } \\
\text { Correlation }\end{array}$} \\
\hline & & & & Side & $\begin{array}{l}\text { Area, } \\
\mathrm{mm}^{2}\end{array}$ & $\begin{array}{c}\text { Thickness, } \\
\text { mm }\end{array}$ & $\begin{array}{l}\text { Area, } \\
\mathrm{mm}^{2}\end{array}$ & $\begin{array}{c}\text { Thickness, } \\
\text { mm }\end{array}$ & \\
\hline 1 & 49 & Male & Absent & Right & 16.5 & 5.9 & 5.2 & 1.5 & Yes \\
\hline 2 & 53 & Male & Partial & Left & 9.0 & 3.6 & 3.2 & 1.0 & No \\
\hline 3 & 30 & Male & Complete & Left & 10.0 & 3.4 & 4.0 & 0.9 & Yes \\
\hline 4 & 45 & Male & Partial & Left & 15.0 & 4.1 & 3.2 & 1.3 & No \\
\hline 5 & 52 & Male & Partial & Right & 6.8 & 3.4 & 4.0 & 1.2 & No \\
\hline 6 & 43 & Male & Complete & Right & 12.8 & 3.3 & 1.9 & 1.3 & Yes \\
\hline 7 & 32 & Male & Complete & Left & 11.7 & 3.3 & 2.0 & 1.3 & Yes \\
\hline 8 & 86 & Male & Partial & Right & 14.7 & 3.9 & 4.9 & 2.2 & No \\
\hline 9 & 53 & Male & Absent & Right & 6.1 & 2.3 & 3.3 & 1.2 & No \\
\hline 10 & 55 & Male & Partial & Left & 8.6 & 3.0 & 3.0 & 1.0 & No \\
\hline 11 & 44 & Male & Complete & Left & 16.3 & 3.2 & 3.2 & 1.3 & Yes \\
\hline 12 & 66 & Male & Complete & Right & 17.2 & 3.5 & 2.7 & 1.2 & Yes \\
\hline 13 & 56 & Male & Complete & Left & 16.6 & 4.3 & 4.2 & 1.6 & Yes \\
\hline Mean & 51 & & Partial (5) & Right (6) & 11.96 & 3.50 & 3.33 & 1.33 & \\
\hline SD & 14 & & $\begin{array}{l}\text { Complete (6) } \\
\text { Absent (2) }\end{array}$ & Left (7) & 3.79 & 0.79 & 1.04 & 0.36 & \\
\hline
\end{tabular}

Figure 5. Images from a 45-year-old man with a partial distal biceps brachii tendon tear and numbness along the lateral forearm. A, Sonogram over the anterior left elbow in the short axis showing an enlarged and echogenic LABCN (white arrows) with loss of the normal fascicular echo texture (cross-sectional area, $15 \mathrm{~mm}^{2}$ ). Note the enlarged distal biceps tendon short head (black arrowhead) and long head (white arrowhead) located just lateral to the $L A B C N$. B, Corresponding long-axis image showing abnormal fusiform thickening and increased echogenicity of the LABCN (solid white arrows; thickness, $4.1 \mathrm{~mm}$ ). Note the transition to normal, first with return of the normal fascicular echo texture (dashed white arrow) and then tapering to normal size and echogenicity (black arrow). The normal nerve is barely perceptible, being isoechoic to slightly hypoechoic to the adjacent subcutaneous fat. C and D, Sonograms of the contralateral normal right elbow in both the transverse $(\mathbf{C})$ and longitudinal (D) planes showing a normal LABCN (white arrows) with preservation of its fascicular echo texture (cross-sectional area, $3.2 \mathrm{~mm}^{2}$; and maximum thickness, $1.3 \mathrm{~mm}$ ). Note the normal distal biceps brachii tendon short head (black arrowhead) and long head (white arrowhead).

A

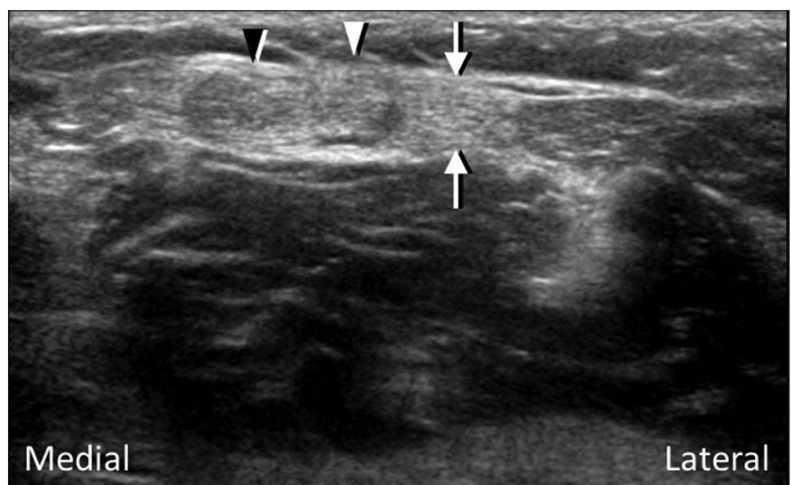

c

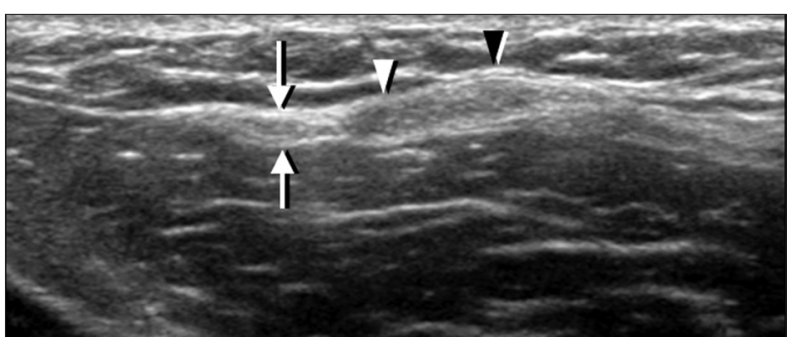

B

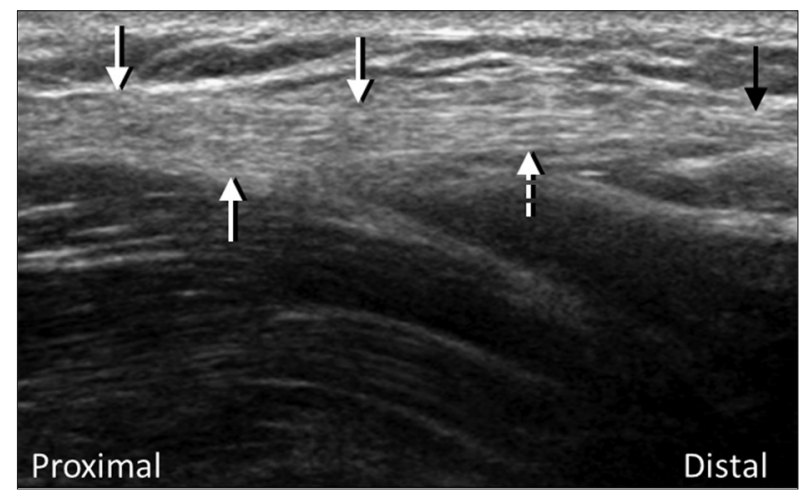

D

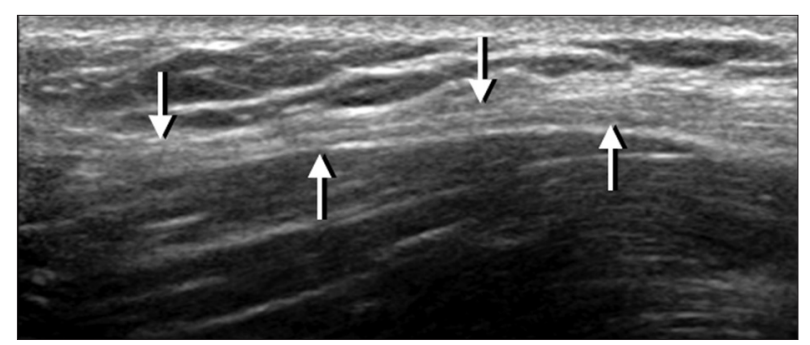


tive abscess after distal biceps brachii tendon repair (Figure 7). In each case, the distal biceps brachii abnormality was adjacent to the abnormal segment of the LABCN.

A review of patient histories revealed that all described symptoms of pain or burning in the LABCN were distributed along the lateral forearm, extending from the elbow to the distal forearm. One patient presented for wrist sonography to "rule out wrist pathology;" however, on further questioning, his pain was mostly within the forearm, radiating to the wrist. Sonography of the elbow revealed an unsuspected distal biceps tendon tear and LABCN abnormality. Wrist sonographic findings were normal. In the 1 patient with an isolated LABCN abnormality in the absence of an adjacent biceps brachii tendon abnormality, there was a history of traumatic venipunctures 5 and 2 months before the sonographic examination, which immediately preceded the onset of forearm symptoms.

\section{Discussion}

The LABCN, also called the lateral cutaneous nerve of the forearm, can be injured or entrapped as a result of various pathologic processes involving the anterior elbow, most commonly distal biceps brachii tendon tears. Understanding the location of this nerve, its relationship to other structures, and its imaging appearance is therefore essential. Our results show that sonography can depict an abnormal LABCN, often related to an adjacent biceps brachii tendon tear, as an explanation for symptoms in the lateral forearm.

The LABCN is a terminal sensory branch of the musculocutaneous nerve, classically described as lying between the biceps brachii and brachialis muscles, emerging approximately $5 \mathrm{~cm}$ proximal to the elbow crease at the lateral edge of the distal biceps brachii myotendinous junction. ${ }^{18,19}$ A later anatomic study found that the LABCN did not emerge proximally but ran along the lateral aspect of the distal biceps brachii tendon until it emerged at the level of the elbow crease (or more distal) to become cutaneous. ${ }^{1}$ Once the nerve becomes subcutaneous, it is sensory only in nature, giving off 1 or 2 trunks and multiple small branches to supply the lateral aspect of the forearm, wrist, and occasionally, the base of the thumb. ${ }^{11,19}$ The LABCN is also closely related to the cephalic vein. Although there is variability in the branching pattern of the veins in the antecubital fossa, the LABCN is located deep to the cephalic vein in most cases. ${ }^{20}$

Injury to the $\mathrm{LABCN}$ can be seen in both the acute and chronic settings. Acute and subacute processes include distal biceps brachii tendon tears, distal biceps brachii tendon repairs, and traumatic cephalic vein phle-
Figure 6. Images from a 44-year-old man with a full-thickness distal biceps brachii tendon tear. A, Sonogram over the anterior left elbow in the transverse plane showing the torn, retracted, and thickened long ( $\mathrm{LH}$; left white arrowhead) and short ( $\mathrm{SH}$; right white arrowhead) heads of the distal biceps brachii tendon with surrounding fluid (black arrowheads). B and $\mathbf{C}$, Sonograms over the anterior left elbow in the transverse (B) and longitudinal (C) planes showing a markedly enlarged and echogenic LABCN (white arrows) surrounded by fluid (black arrowheads) with loss of the normal fascicular echo texture (cross sectional area, $16.3 \mathrm{~mm}^{2}$; maximum thickness, $3.2 \mathrm{~mm}$ ). C indicates cephalic vein.

A

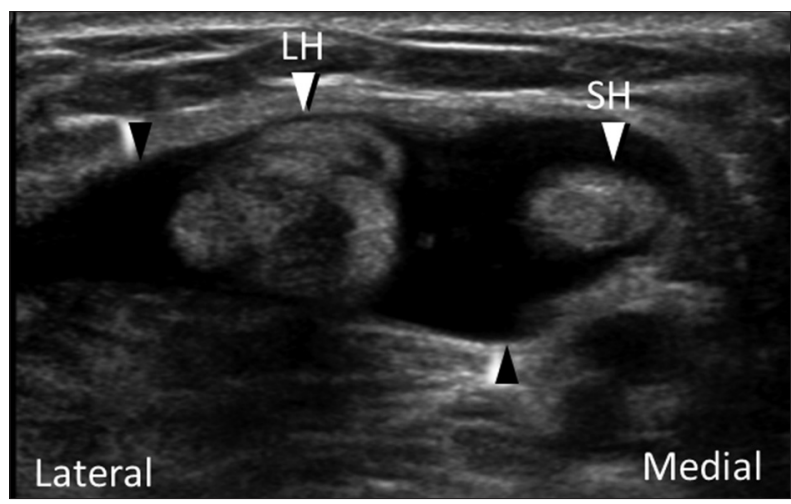

B

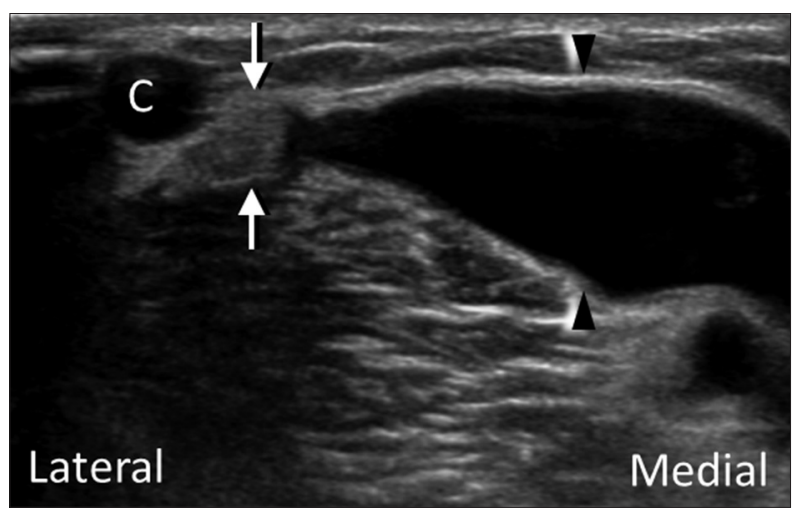

c

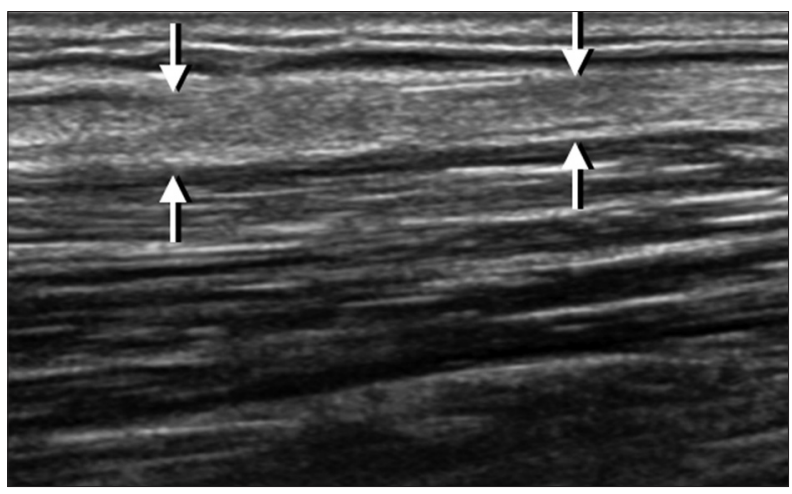


botomy, given the close proximity of the $\mathrm{LABCN}$ to these structures. ${ }^{5-7,10}$ The LABCN can also be entrapped secondary to fractures that involve the distal humerus, proximal radius, or olecranon process of the ulna. This condition may occur from direct compression by hematoma or may be triggered by cast placement with compression of the lateral antebrachial fascia, fibrous band formation, and constriction of the $\mathrm{LABCN}{ }^{4}$ The $\mathrm{LABCN}$

Figure 7. Images from a 49-year-old man with chronic infection after a distal biceps brachii tendon repair. A, Transverse sonogram over the anterior elbow showing a markedly thickened and echogenic LABCN (white arrows; cross sectional area, $16.5 \mathrm{~mm}^{2}$ ) adjacent to a thickened distal biceps brachii tendon (white arrowheads). B, Long-axis sonogram showing the enlarged and echogenic LABCN (white arrows; maximum thickness, 5.9 $\mathrm{mm}$ ). C, Color Doppler sonogram in the transverse plane showing hyperemia of the inflamed distal biceps brachii tendon (white arrowheads) with intraneural vascularity of the LABCN (white arrows). D and E, Axial short-tau inversion recovery (D) and contrast enhanced T1-weighted fat-saturated (E) MRI showing an enlarged LABCN (yellow circles) adjacent to a thickened distal biceps brachii tendon (blue arrows) surrounded by complex enhancing fluid. Br indicates brachialis muscle; and BR, brachioradialis muscle. F, Sonogram of the contralateral asymptomatic right elbow in the transverse plane showing a normal hypoechoic $\mathrm{LABCN}$ (white arrows; cross sectional area, $5.2 \mathrm{~mm}^{2}$ ). White arrowhead indicates distal biceps brachii tendon.

A

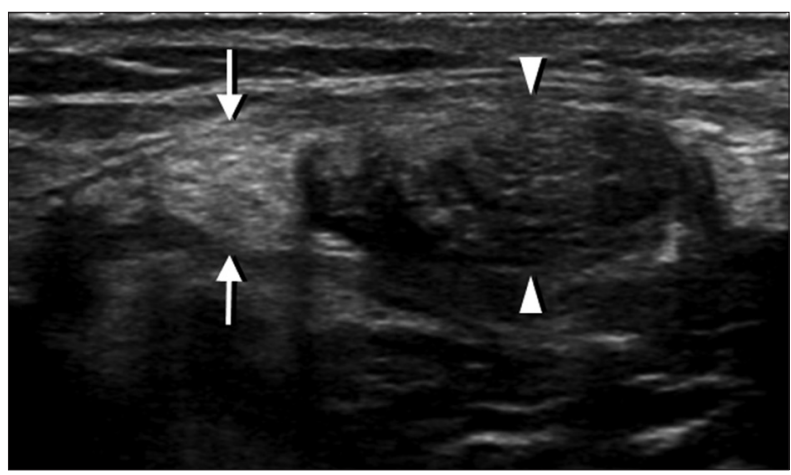

C

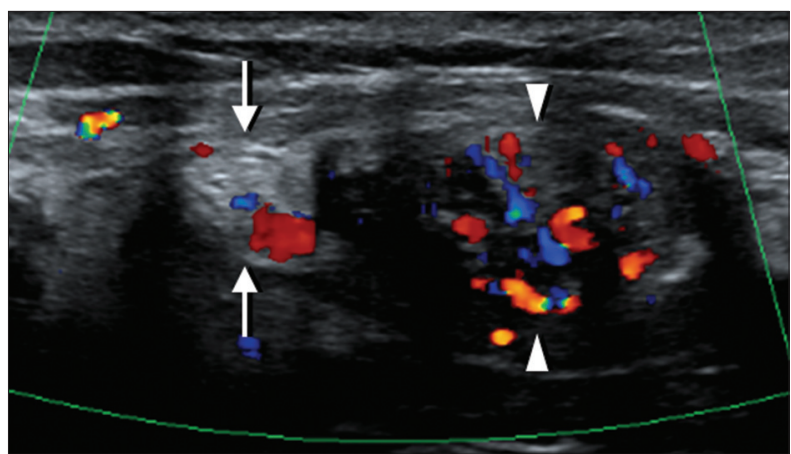

E

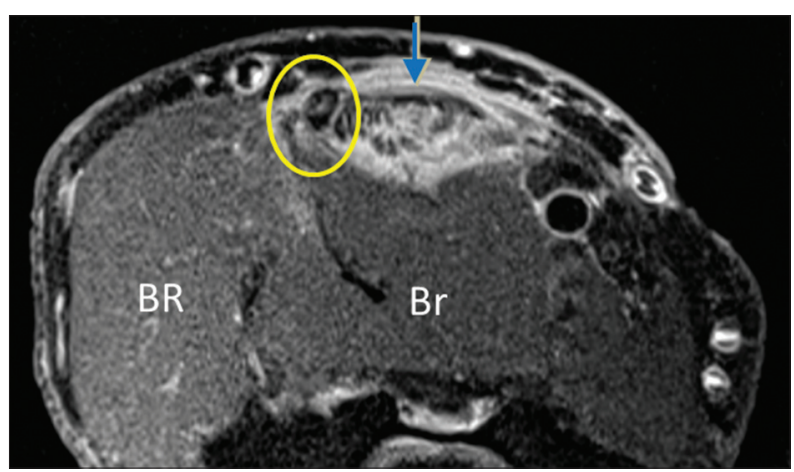

B

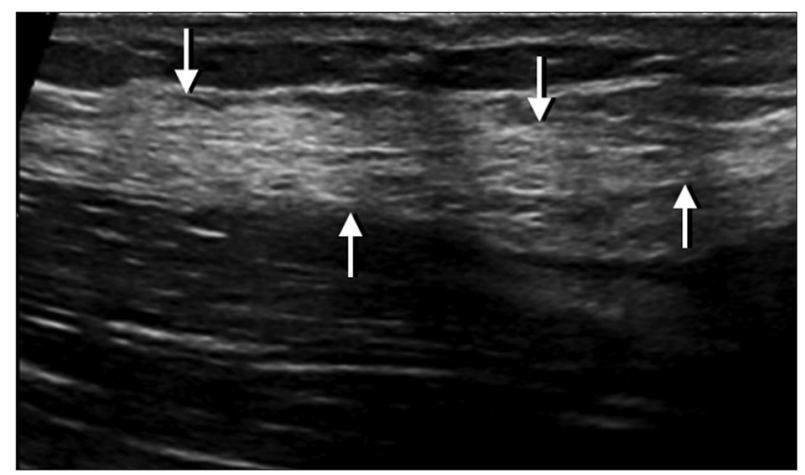

D

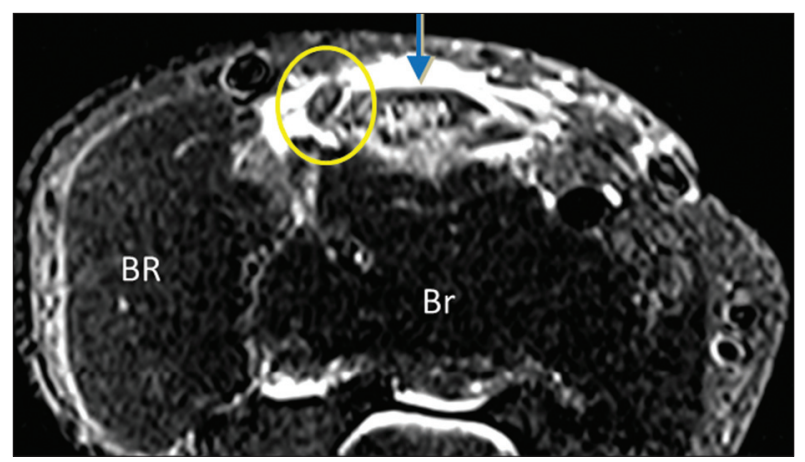

$\mathrm{F}$

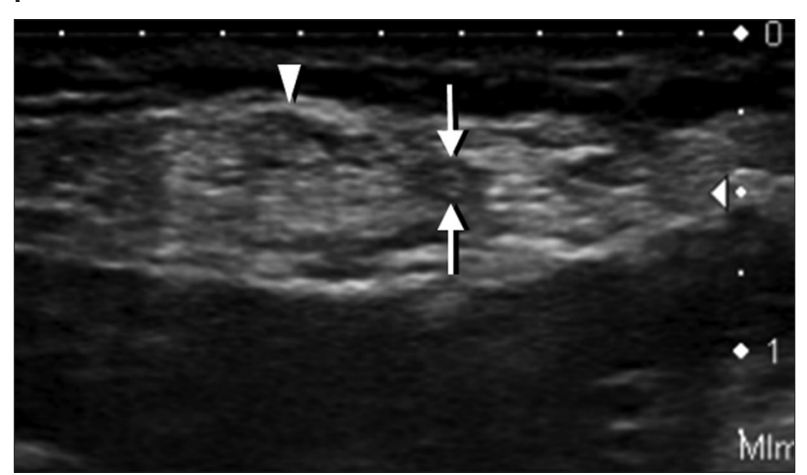


may be chronically impinged by various repetitive movements requiring repeated pronation and supination with the arm fully extended, which can be more difficult to diagnose, often being confused with lateral epicondylitis or "tennis elbow." 3 When the arm is fully extended and maximally pronated, the sharp lateral free edge of the bicipital aponeurosis can cause direct compression of the nerve. Lateral antebrachial cutaneous nerve injury has also been described in patients after a fall on an outstretched hand with the elbow extended, in the absence of a fracture, due to acute compression by the lateral free margin of the bicipital aponeurosis. ${ }^{3}$ In the acute setting, patients typically describe pain and burning dysesthesia in the lateral forearm, radiating distally, whereas in chronic impingement, patients describe vague, aching discomfort in the antecubital region and may be unaware of the numbness in the forearm or hand. ${ }^{3}$

On sonography, a normal peripheral nerve when imaged in cross section has a honeycomb or fascicular appearance with hypoechoic nerve fascicles and surrounding hyperechoic connective tissue and epineurium. ${ }^{17}$ Sonography of the normal LABCN in the cadaveric specimen and the contralateral elbows of the patients in our study showed a similar appearance.

In each of our patients with an abnormal LABCN, a segment of the LABCN was enlarged, with increased echogenicity and loss of the fascicular pattern compared to the contralateral asymptomatic side. When evaluating a potential peripheral nerve abnormality, comparison to the contralateral side has been shown to be reliable. ${ }^{21}$ In our patients with a distal biceps brachii tendon abnormality (tear or infected fluid collection), the abnormal segment of the LABCN was seen immediately adjacent. The single case without an adjacent abnormality had a history of traumatic venipuncture. In all cases, the abnormally enlarged $\mathrm{LABCN}$ was increased in echogenicity, with loss of the normal fascicular pattern compared to the contralateral side. The cause of the increased echogenicity is uncertain. The typical appearance of entrapment neuropathy is that of hypoechoic nerve enlargement. ${ }^{22}$ Similarly, a traumatic neuroma will appear hypoechoic on sonography. ${ }^{23}$ It is possible that edema or hemorrhage in and around the LABCN causes an increase in the number of interfaces and an increase in echogenicity. Indeed, in each of our patients with MRI, there was an increased fluid signal in and around the LABCN, which supports this hypothesis.

We acknowledge some limitations to this study, which include the limited number of patients and a single cadaveric specimen, although to our knowledge, this study is the first to present a series of patients with LABCN abnormalities. In addition, normative values of the $\mathrm{LABCN}$ were not determined; however, comparison to the contralateral asymptomatic side has been shown to be reliable as an internal control for detection of upper extremity nerve abnormalities. ${ }^{21}$ Last, the true incidence of LABCN abnormalities, either in isolation or associated with other disorders such as distal biceps brachii tendon abnormalities, could not be assessed because of the retrospective nature of this study, where the LABCN may not have been routinely evaluated or imaged prospectively, which would not allow later characterization.

In summary, we have shown that $\mathrm{LABCN}$ abnormalities are characterized by symmetric fusiform thickening and increased echogenicity with loss of the normal fascicular echo texture, often associated with distal biceps brachii tendon abnormalities or possible traumatic venipuncture. Knowledge of the location of the LABCN, possible association with adjacent abnormalities, and symptoms referable to the lateral forearm are important for accurately diagnosing the cause of patients' symptoms.

\section{References}

1. Olson IA. The origin of the lateral cutaneous nerve of forearm and its anaesthesia for modified brachial plexus block. J Anat 1969; 105:381382.

2. Naam NH, Massoud HA. Painful entrapment of the lateral antebrachial cutaneous nerve at the elbow.J Hand Surg Am 2004; 29:1148-1153.

3. Bassett FH III, NunleyJA. Compression of the musculocutaneous nerve at the elbow. J Bone Joint Surg Am 1982; 64:1050-1052.

4. Belzile E, Cloutier D. Entrapment of the lateral antebrachial cutaneous nerve exiting through the forearm fascia.J Hand Surg Am 2001; 26:64-67.

5. Brogan DM, Bishop AT, Spinner RJ, Shin AY. Lateral antebrachial cutaneous neuropathy following the long head of the biceps rupture.J Hand Surg Am 2012; 37:673-676.

6. Maak TG, Osei D, Delos D, Taylor S, Warren RF, Weiland AJ. Peripheral nerve injuries in sports-related surgery: presentation, evaluation, and management. AAOS exhibit selection. J Bone Joint Surg Am 2012; 94:e1211e1210.

7. Cain RA, NydickJA, Stein MI, Williams BD, Polikandriotis JA, Hess AV. Complications following distal biceps repair. J Hand Surg Am 2012; 37:2112-2117.

8. Sander HW, Conigliari MF, Masdeu JC. Antecubital phlebotomy complicated by lateral antebrachial cutaneous neuropathy. NEngl J Med 1998; 339:2024

9. Stevens RJ, Mahadevan V, Moss AL. Injury to the lateral cutaneous nerve of forearm after venous cannulation: a case report and literature review. Clin Anat 2012; 25:659-662.

10. Stitik TP, Foye PM, Nadler SF, Brachman GO. Phlebotomy-related lateral antebrachial cutaneous nerve injury. Am J Phys Med Rehabil 2001; 80:230-234. 
11. WongkerdsookW,Agthong S,Amarase C, YotnuengnitP,Huanmanop T, Chentanez V. Anatomy of the lateral antebrachial cutaneous nerve in relation to the lateral epicondyle and cephalic vein. Clin Anat 2011;24:56-61.

12. Rayegani SM, Azadi A. Lateral antebrachial cutaneous nerve injury induced by phlebotomy. J Brachial Plex Peripher Nerve Inj 2007;2:6.

13. Judge A, Fecho K. Lateral antebrachial cutaneous neuropathy as a result of positioning while under general anesthesia. Anesth Analg 2010; 110:122-124.

14. Yildiz N, Kilinc B, Ardic F. A soldier with lateral antebrachial cutaneous neuropathy: malingering or fact? TurkJPhys Med Rehabil 2010; 56:145147.

15. Tagliafico AS, MichaudJ, Marchetti A, Garello I, Padua L, Martinoli C.US imaging of the musculocutaneous nerve. Skeletal Radiol 201 1; 40:609616.

16. Kotnis NA, Chiavaras MM, Harish S. Lateral epicondylitis and beyond: imaging of lateral elbow pain with clinical-radiologic correlation. Skeletal Radiol 2012; 41:369-386.

17. Silvestri E, Martinoli C, Derchi LE, Bertolotto M, Chiaramondia M, Rosenberg I. Echo texture of peripheral nerves: correlation between US and histologic findings and criteria to differentiate tendons. Radiology 1995; 197:291-296.

18. de Jong RH. Axillary block of the brachial plexus. Anesthesiology 1961; 22:215-225.

19. de Jong RH. Modified axillary block with block of the lateral antebrachial cutaneous (terminal musculocutaneous) nerve. Anesthesiology 1965; 26:615-618.

20. Mikuni Y, Chiba S, Tonosaki Y. Topographical anatomy of superficial veins, cutaneous nerves, and arteries at venipuncture sites in the cubital fossa. Anat Sci Int 2013; 88:46-57.

21. Tagliafico A, Martinoli C. Reliability of side-to-side sonographic crosssectional area measurements of upper extremity nerves in healthy volunteers. J Ultrasound Med 2013; 32:457-462.

22. Jacobson JA, Fessell DP, Lobo Lda G, Yang LJ. Entrapment neuropathies I: upper limb (carpal tunnel excluded). Semin Musculoskelet Radiol 2010; 14:473-486.

23. Tagliafico A, Altafini L, Garello I, Marchetti A, Gennaro S, Martinoli C. Traumatic neuropathies: spectrum of imaging findings and postoperative assessment. Semin Musculoskelet Radiol 2010; 14:512-522. 